\title{
Dog Dander Antigen IgE Antibody Measurement
}

National Cancer Institute

\section{Source}

National Cancer Institute. Dog Dander Antigen IgE Antibody Measurement. NCI

Thesaurus. Code C130128.

A measurement of the dog dander antigen IgE antibody in a biological specimen. 